\title{
STATISTICAL ANALYSIS OF 100 BREAST CANCER CASES WITH EVALUATION OF HER2/NEU STATUS IMMUNOHISTOCHEMISTRY CORRELATION WITH GENE AMPLIFICATION BY FLUORESCENCE IN SITU HYBRIDIZATION ASSAY
}

\author{
Sivakami1, Indhu Kannan², Madhusudhanan ${ }^{3}$
}

${ }^{1}$ Senior Consultant, Department of Pathology, Meenakshi Mission Hospital and Research Centre, Madurai, Tamilnadu, India. ${ }^{2}$ Consultant Pathologist, Department of Pathology, Meenakshi Mission Hospital and Research Centre, Madurai, Tamilnadu, India. ${ }^{3}$ Consultant Pathologist, Department of Pathology, Meenakshi Mission Hospital and Research Centre, Madurai, Tamilnadu, India.

ABSTRACT

\section{BACKGROUND}

Breast cancer has surpassed all other cancers in women becoming the leading cause for mortality. 100 breast cancer cases received in our department of pathology during the period 2016-2017 were evaluated for HER2/neu status and immunohistochemistry correlation with gene amplification by FISH assay.

\begin{abstract}
MATERIALS AND METHODS
Mastectomy samples received were fixed followed by grossing including all parameters like laterality, size, margin status and adequate lymph node sampling. The tissue sections were stained with $\mathrm{H} \& \mathrm{E}$ for assessing the tumour type, grade, surgical margin clearance study. Lymph node involvement, oestrogen receptor and progesterone receptors Her2/neu was done with immunohistochemistry in the tumour blocks.
\end{abstract}

Subsequently tissue blocks were also subjected to FISH evaluation for Her2/neu.

\section{RESULTS}

The tumour laterality, tumour categorization, staging, and grading were analysed. Comparison of immunohistochemical Her2 results with FISH was done.

\section{CONCLUSION}

Immunohistochemical analysis of Her2/neu with 0 and $1+$ Negative. $3+$ strong positive had a High concordance with FISH evaluation.

\section{KEY WORDS}

Breast Cancer, Infiltrating Ductal Carcinoma (NOS) HER 2 Gene Immunohistochemistry Fluorescence, in Situ Hybridization.

HOW TO CITE THIS ARTICLE: Sivakami, Kannan I, Madhusudhanan. Statistical analysis of 100 breast cancer cases with evaluation of her2/ neu status immunohistochemistry correlation with gene amplification by fluorescence in situ hybridization assay. J. Evolution Med. Dent. Sci. 2018;7(49):5298-5303, DOI: 10.14260/jemds/2018/1174

\begin{abstract}
BACKGROUND
Breast cancer accounts for $27 \%$ of all cancers and is the cause of $16 \%$ cancer deaths. In the western population, incidence increases with age when compared to the Asian population. The human epidermal growth factor receptor 2 (Her2/ neu) or (ErB-2) gene is a member of ErbB family of receptor and is located in human chromosome 17q21. The Her2 gene ErbB2 gene was called NEU to begin with because it was first derived from rat neuroblastoma/ glioblastoma cell lines. Subsequently Okita and colleagues ${ }^{1}$ named it Her2 because the primary sequence was similar to human epidermal growth factor receptor (EGFR, ERBB or ERBB1) Semba and associates independently identified an ERBB related but distinct gene which they named ERBB2. Di Fiore and colleagues indicated that both NEU and HER2 were the same as ERBB2, and Akiyama and associates precipitated the
\end{abstract}

'Financial or Other Competing Interest': None.

Submission 07-09-2018, Peer Review 16-11-2018,

Acceptance 23-11-2018, Published 03-12-2018.

Corresponding Author:

Sivakami,

Pathology Laboratory Services,

Meenakshi Mission Hospital and Research Centre,

Lake Area, Melur Main Road,

Madurai-625107, Tamilnadu, India.

E-mail: mmhrcpathology@gmail.com

DOI: $10.14260 /$ jemds $/ 2018 / 1174$
ERBB2 gene product from adenocarcinoma cells and demonstrated it to be a $185-\mathrm{kD}$ glycoprotein with tyrosine kinase activity.

The availability of trastuzumab recombinant humanized mouse monoclonal antibody directed against the Her2 NEU protein for management of metastatic breast cancer has created significant therapeutic implications for breast cancers with Her2 NEU over expression.

Favorable clinical response with trastuzumab was observed in patients with HER-2/neu amplification, however, requires accurate determination of HER-2/neu status.

Several techniques like immunohistochemistry (IHC) on paraffin-embedded tissues for HER-2/neu protein detection, Southern blot analysis, a reverse transcription-polymerase chain reaction (RT-PCR) technique chromogenic in situ hybridization (CISH) technique and fluorescence in situ hybridization (FISH) to quantify HER2/neu are now available. HER-2/neu testing is used as a prognostic marker to determine the aggressiveness of breast cancer tumour. To monitor cancer treatment serum Her2/ NEU level is sometimes evaluated to establish a baseline and if it is elevated is useful as prognostic factor. However, HER2/NEU levels are elevated only when the tumour is large and is in an advanced stage. Early cancers are likely to be Negative for serum HER2/NEU. FISH has become an increasingly popular assay and is being widely used for diagnosis as well as to monitor the response to therapy, such as hormone therapy 
and chemotherapy. In this study we investigated HER$2 /$ neu gene amplification by FISH in breast carcinoma specimens and compared the results of FISH analysis with IHC. 2

HER2 positive tumour also generally shows resistance to most of the current available endocrine therapies. Most importantly HER2 targeted therapies are available which has made this biomarker the most important theranostic testing for breast cancer. The most common methods currently used for determining the HER2/NEU in breast cancers are immunohistochemistry (IHC measuring protein level) and Fluorescence in situ hybridization FISH (measuring the gene level).

Improper fixation and poor processing techniques may alter the antigenic expression and may produce false positive or false negative results in IHC evaluation for HER2.

FISH on the other hand has an excellent sensitivity and specificity in detecting HER2 gene amplification. One of the major limitations for FISH evaluation of HER2 is its relative high cost and requirement of special instruments.

\section{MATERIALS AND METHODS}

\section{Specimen Collections}

The descriptive study was done in 100 mastectomy specimen samples received in our Meenakshi Mission Hospital and Research Centre Madurai.

The patients had not been subjected to prior chemotherapy or Radiotherapy.

The specimens where fixed in 10\% neutral buffered formalin according to our standard fixation procedures. Tissue processing paraffin embedding done as for other tissue samples. Microscopic diagnosis of infiltrating ductal carcinoma was confirmed with the representative sections and were subsequently subjected to Estrogen Receptor Progesterone receptor and HER2/ NEU status evaluation.

Immunohistochemistry

The polyclonal antigen (Primary) for ER, PR and HER2/NEU antibody was purchased from biogenex and path in situ. Our own positive and negative control sections where run for the evaluation. All IHC staining was performed at room temperature in automated machine.

Rotary microtome was used to take sections 3-5 $\mu \mathrm{m}$ thin. Xylene and ethanol used for deparaffinisation. Sections were immersed in fresh $3 \%$ hydrogen peroxide to inhibit endogenous tissue peroxidase activity for 10 minutes and rinsed with deionised water. The tissue sections were subjected to pressure cooker antigen retrieval method in citrate buffer, $\mathrm{pH}$ 6.8. Cooling time of 20 minutes given to the slides subsequently rinsed thoroughly in the buffer. Blocking serum solution was then used for incubating the sections for 5-10 minutes. Further incubation for 15 minutes done with primary antibody. Washing done with buffered agent. First incubation was done with biotinylated secondary antibody solution done for 25 minutes. 15 minutes with horseradish peroxidase conjugated streptavidin biotin complex done. Subsequently the sections were washed in buffer solution. Freshly prepared mixture of diaminobenzidine (DAB) incubation for 5 minutes 2 changes done which is the most vital step. Then the final step of counter staining done with hematoxylin.
Graded series of ethanol and xylene were used for dehydration and finally the slides are ready for mounting with coverslips.

Two pathologists viewed the slides with light microscope for unbiased opinions.

Results where then documented.

Positivity for HER2 expression was identified by membrane positivity with brown granular color condensation. Grading done from 0-3 based on the proportion of cells positively stained and also based on the degree of intensity.

0 - No membrane staining or faint staining in less than $30 \%$ of tumour cells.

$1+$ incomplete and or faint membrane staining in more than $30 \%$ of tumour cells.

$2+$ incomplete and or weak to moderate membrane staining circumferentially within more than $30 \%$ of tumour cells or intense circumferential membrane staining within less than $30 \%$ of tumour cells. $3+$ intense and complete circumferential membrane staining more than $30 \%$ of tumour cells.

Negative staining was 0 and $1+, 2+$ was graded as equivocal and $3+$ as positive HER2 over expression.

\section{RESULTS}

In our study, the mean age of patients at diagnosis was $54 \mathrm{yrs}$. (Range 35-82 yrs.) 100\% of cases were females. Oldest patient was 82 yrs. old and the youngest 35 yrs. Among 100 cases 49 cases had left side disease 51 cases had right side disease.

Lymph node metastasis was found in 59 cases.

Histological grade was I, II, III in 4, 61 and 35 cases respectively.

Size of tumour was T1 $(0-2 \mathrm{cms})$ in 5 cases.

T2 (2-5 cms) in 65 cases, T3 more than $5 \mathrm{cms}$ in 18 cases.

T4b (Tumour involving skin) was identified in 12 cases.

Estrogen receptor positivity was detected in 51 cases.

Progesterone receptor positivity was detected in 48 cases.

12 cases were classified as IHC $2+$ weak positive, 21 cases as IHC $3+$ strong Positive and 67 cases were IHC 0 and $1+$ Negative.

\section{IHC and Fish HER2 Comparison}

FISH was used as gold standard when comparing IHC and FISH results.

Highest concordance rate of (98\%) was seen in the IHC negative 0 and $1+$.

High concordance of $95 \%$ was observed in IHC 3+ strong positive. IHC 2+ Weak Positive had a Concordance of $35 \%$.

\section{HER2 Status Correlation with Patient's Parameter}

Various histopathological parameters like tumour size, age, lymph node metastasis were analyzed with HER2 over expression and there was found to be no correlation with these parameters and HER2 expression in our study.

$61 \%$ of FISH positive Her2 with $2+$ Weak positive with IHC were grade III tumours in our study. 


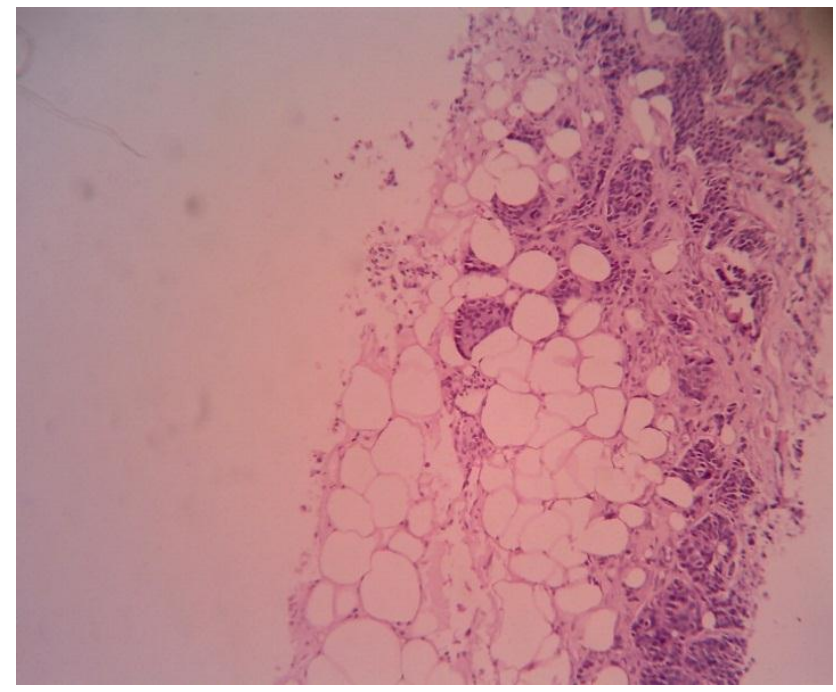

Figure 1. Infiltrating Ductal Carcinoma Low Power View

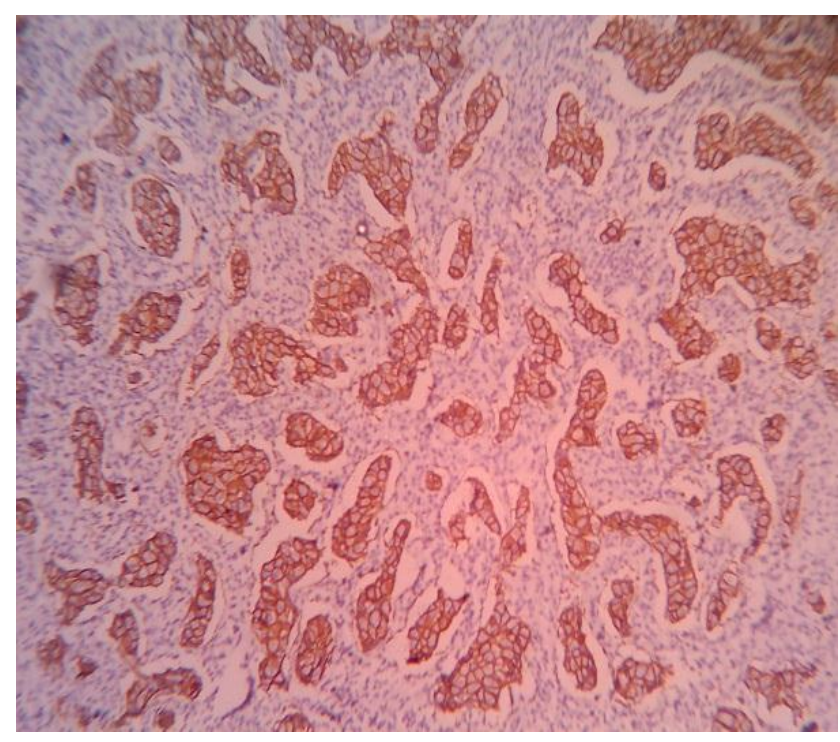

Figure 2. IHC Her2/neu 3+ Strong Positive Low Power View

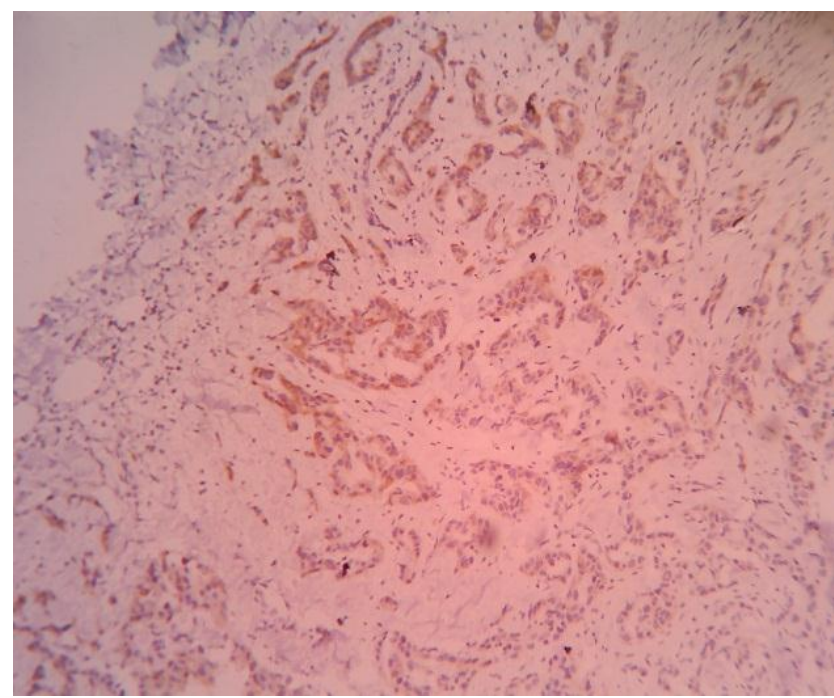

Figure 3. IHC Her2/neu 2+ Weak Positive FISH Positive Low Power View

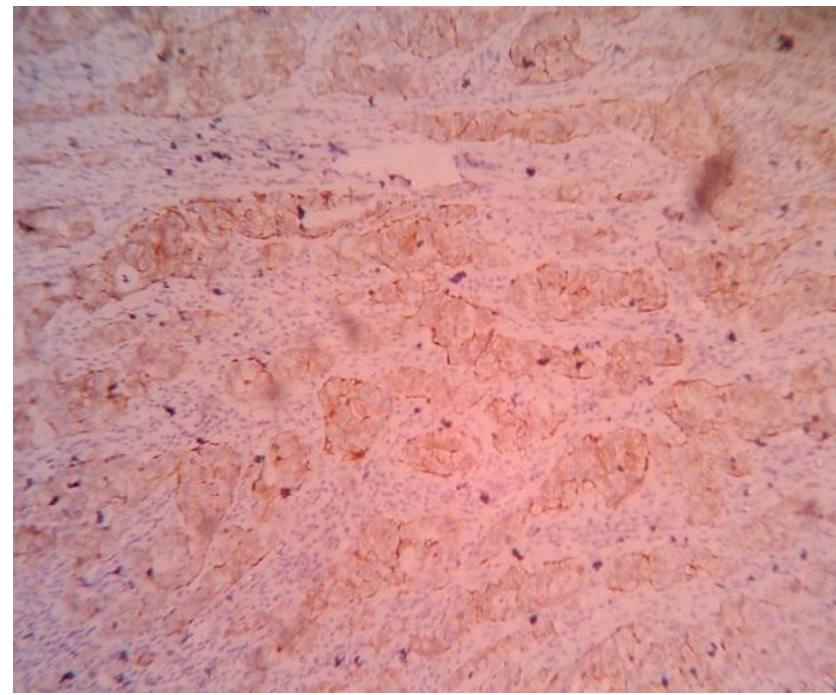

Figure 4. IHC Her2/neu 2+ Weak Positive FISH Negative Low Power View

\section{DISCUSSION}

Salmon et $\mathrm{al}^{3}$ published the importance of HER2/ NEU as poor prognostic factor in 1987. Since then the relevance of HER-2/NEU oncogene amplification in breast cancer has gained momentum and has proved its significance in planning treatment protocols. Likewise, it has been implicated as a prognostic factor in many other cancers, as well, such as ovary, lung, stomach, and pancreas.

Evaluation of HER2/NEU with reliable laboratory data is of utmost importance since patients treated with combination of antineoplastic agents like anthracyclines and trastuzumab alone are prone to develop cardiotoxic side effects. 4

We evaluated in our study the concordance of Her-2/neu expression in 100 infiltrating breast carcinoma specimens by FISH and IHC. The US FDA recommends and approves IHC and FISH methods for evaluation of HER2 neu status of these two methods IHC is recommended as screening test and FISH as the gold standard for detecting HER2 status by NCCN (National Comprehensive Cancer Network)

Our results revealed that Statistical analyses showed that both the assays are related to each other, supporting the validity of these tests. In clinical lab settings IHC is simple and relatively inexpensive test to perform many issues that interfere with optimization and proper assessment still exist. The foremost concern would be background staining as Her$2 /$ neu is expressed in normal epithelial cells as well. Critical steps including proper handling of the specimen, appropriate fixation, paraffin block preparation, antigen retrieval also have a serious impact on the test results. Also, there are numerous anti-Her-2/neu antibodies, that are available with varying sensitivities and specificities. Some interlaboratory discrepancies may exist for at least some of the cases, especially the weak positive ones. Hence this type of inconsistency could pose difficulties in planning Herceptin treatment.

FISH is at present the most effective procedure for quantitation of gene duplication to determine H2N status in breast cancers and other cancers. 
Formalin fixed paraffin embedded tissue is used to asses H2N status by FISH.

Many factors like fixation of tissues sectioning artifacts may interfere in quantification of signals and interpretation.

DNA being very stable, is less susceptible to variations in tissue fixation processing thus, providing optimal correlation between morphology and hybridization findings.

Since IHC measures protein concentrations and FISH assays measure gene copies, some discordance is to be expected, when there is a high concentration of the Her$2 /$ neu protein without concurrent gene amplification.

In our study, the concordance for IHC 0 and $1+$ negative was $98 \%$. Concordance for IHC 3+ Strong positive was $95 \%$. IHC 2+ Weak positive had a concordance of 35\%.

Most of the Published reports have documented that the $1+$ and $2+$ categories are the most difficult to assess and FISH should be considered in these cases. (Ledeau and Perez et al)5,6. A case study by Aziza et ${ }^{7}$ al have reported that the discordance between IHC and FISH results, for these categories is due to chromosome 17 Aneuploidy.

Some clinical trials have also proved that FISH techniques in assessing HER2/NEU status to be superior to assess the predictive response to trastuzumab when compared to IHC. 8

Studies which have adopted similar strategy have been reported. Dolan et al have published work on studying the interobserver variability in immunohistochemical scoring, in which multiple pathologists submit specimens to a single cytogenetics referral laboratory. They found a higher rate of discordance between immunohistochemical analysis and FISH (Approximately 92\%) in the groups with immunohistochemical scores of $2+$ than reported in other studies.

Worthy of mention, a Study by Jacob et $\mathrm{al}^{9}$ compared the results from 2 laboratories for HER-2/neu determined by IHC on paraffin sections of the same 100 consecutive invasive breast cancers. They ensured that both laboratories used the same primary antibody; however, the specimens were subjected to different retrieval methods and scoring systems. They were able to achieve complete concordance in categorization of HER-2/neu status between the 2 laboratories and proved excellent interlaboratory agreement for HER-2/neu IHC was attainable using the same primary antibody to HER-2/neu, even without optimization or scoring criteria.

Study by Sui et al showed that different sensitivity and specificity of the antibodies used in FISH protein may lead to discordance in the two assays as their target portion of the protein molecule is different. Further research would be needed to align the target focus to improve the sensitivity of the tests.

In 2+ Weak positive and negative cases when the tumour grade is high. Kuo et al ${ }^{10}$ proposed an algorithm in which IHC is used first to screen the HER2 status and FISH may be then used as supplementary test to asses HER2 status. Herceptin therapy also was recommended only for $3+$ strong positive HER2 cases or FISH positive cases.

Park et al11, in addition, emphasized that apart from IHCequivocal cases, FISH assay is to be considered for a selected group of patients with IHC-negative tumours showing luminal B subtype features of ER-positive, grade II/III, and high Ki-67 expression.
The prevalence of ERb - b2 gene amplification 2+ Positive IHC cases was studied by Lan et al and included 221 patient samples that majorly comprised of metastatic and recurrent breast cancer cases. The study revealed $44.4 \%$ of the samples to be erb-b2 amplified. In addition, the topoisomerase IIa gene was coamplified in $21 \%$ and deleted in $15 \%$ of the erbb2 amplified cases. The erb-b2 gene amplification rate was very high in cases determined to be $2+$ by immunohistochemical analysis; therefore, suggested that the determination of erb-b2 status by FISH in cases scored 2+ immunohistochemically is strongly recommended.

With more studies in consideration, an agreement between IHC and FISH is still controversial. The concordances in the cases of IHC $3+$ are similar but there is a difference in the cases of IHC $2+$.

False IHC results may be due to various factors like preanalytical variable issues including tissue fixation and processing. Differing sensitivity and specificity of the available commercial antibodies. Interobserver variations. Differences in scoring criteria in interpretation of results or improper IHC methodology.

Comparing studies in literature using FISH is difficult because of the differences in criteria in defining amplification these include the ratio between gene copies comparing chromosome 17 copies, and an absolute number of gene copies, and the mean number of copies. Studies of FISH have been carried out on frozen section samples and touch preparations as well. Irrespective of the sample used, most of the studies have agreed that FISH is a rapid and reproducible method that allows the accurate measurement of the level of oncogene amplification within interphase nuclei ${ }^{12}$. Attempts to correct variability of criteria reflects in correction for artifacts, as well as intrinsic tumour heterogeneity.

Some studies have proposed the combination of in situ hybridization and immunohistochemical techniques to evaluate all degrees of amplification of the oncogene Her2/neu and overexpression of its protein c-erbB-2. This permits the simultaneous evaluation of the oncogene and its corresponding protein expression in single cells and specific cellular populations in histologic tissue sections. Double staining demonstrated heterogeneity within breast carcinomas. In addition, both nuclear and cytoplasmic signals were often detected in morphologically normal-appearing adjacent breast epithelium. The ability to view both the oncogene and its corresponding protein in single cells offers a unique look at the biology of c-erbB-2.13.

Strong H2N immunostaining is highly associated with gene amplification as advocated by Rafeal ${ }^{14}$ et al although there is minor variation in sensitivity among different antibodies which are used for IHC. They also suggested that correlation with FISH data may be necessary to optimize interpretation of IHC stains for standardization of the technique. Gene amplification, as detected by FISH, is usually associated with a high number of gene copies per nucleus rather than cytoplasmic or membranous staining which was also suggested in the studies. Low-grade duplication of $\mathrm{H} 2 \mathrm{~N}$ gene ( 3 to 4 copies/cell) is usually the result of chromosome 17 polysomy and is not usually in association with high-level overexpression. Also, some light over her 2 neu status in DCIS they, suggested that overexpression without amplification may be more frequent in cases of DCIS than infiltrating 
carcinomas raising the questions about different roles of $\mathrm{H} 2 \mathrm{~N}$ in the biology of preinvasive and invasive neoplasm.

The clinical implications of HER-2/neu (HER2) expression in ductal carcinoma in situ (DCIS) lesions are yet to be clearly elucidated. Many studies have compared HER2 in expression in high-grade DCIS lesions with invasive cancers and have revealed data that strengthen the argument that HER2 overexpression in DCIS is associated with more rapid progression to invasive disease. Thus, HER2 overexpression in DCIS lesions predicts the presence of invasive foci in patients with DCIS and suggest that targeting of HER2 in an early disease setting may forestall or prevent disease progression ${ }^{15}$. We encountered 4 cases of DCIS in our study period, but the patients were lost on follow up.

Several studies revealed the relationship of expression between subtypes (ER, PR and HER2) with age. Most suggested higher expression of ER and PR in female who were 50 or younger ${ }^{16}$. There were no significant differences between the subtypes regarding age in present study.

Another similar study conducted to analyze the association between the expressions of eight immunohistochemical (IHC) indices including Her2 neu and its correlation with clinicopathological characteristics in breast cancers (BCs) and investigate the clinical significance in 286 cases of invasive BC and statistically analyzed their correlations with clinicopathological characteristics. Their data revealed that Luminal B and HER2/neu subtypes exhibited a close correlation with age, while the HER2/neu and triple-negative subtypes were positively correlated with poor histological grade. ${ }^{17}$

\section{CONCLUSION}

In various studies, a high concordance is documented between IHC 0/1+ and 3+ with FISH hence IHC alone can be used as screening test to evaluate HER2 status in these cases. There is a poor concordance between IHC $2+$ and FISH hence in the 2+ weak positive cases, it is advised to perform FISH test in order to exclude wrong assessment of HER2 status. ${ }^{18}$ In summary, our study provides data on stable HER2 positivity-rate determined by FISH technology on a consecutive diagnostic breast cancer cohort and explores how concordance-rate between IHC and FISH technology can be improved by applying standardized analytical and pre-analytical procedures.

The question as to whether there is a best methodology as the gold standard for HER2 testing remains controversial as FISH has some drawbacks as well. Whatever methodology is used in the given pathology institution, standardized preanalytical, analytical and scoring issues need to be guaranteed in order to provide accurate HER2 test results in the diagnostic setting.

In our study, the association between these indices and the clinicopathological characteristics of breast cancers was mostly similar to other related previous studies.

In our analysis as well, we also found that age of patients' laterality, size of tumour or nodal status had no correlation with Her2 status. However, we feel that a larger cohort study is needed to prove or disprove definite correlation between clinicopathological characteristics of breast cancers and the IHC indices. In the evaluation of biological behavior and prognosis of breast cancers, combined detection of these indices may play a vital and significant role for assessment of prognostic factors and also in the comprehensive treatment of this disease.

\section{REFERENCES}

[1] Okita Y, Narita Y, Suzuki T, et al. Extended trastuzumab therapy improves the survival of HER2 positive breast cancer patients following surgery and radiotherapy for brain metastases. Mol Clin Oncol 2013;1(6):995-1001.

[2] Song Q, Huang R, Li J, et al. The diverse distribution of risk factors between breast cancer subtypes of ER, PR and HER2: a 10 - year retrospective multi - center study in China. PLoS One 2013;8(8):e72175.

[3] Salmon DJ, Godolphin W, Jones LA, et al. Studies of the HER - 2/neu proto - oncogene in human breast and ovarian cancer. Science 1989;244(4905):707-12.

[4] Keefe DL. Trastuzumab-associated cardiotoxicity. Cancer 2002;95(7):1592-600.

[5] Ledeau A, Deimling D, Kaltz C, et al. Her-2/neu analysis in archival tissue samples of human breast cancer: comparison of immunohistochemistry and fluorescence in situ hybridization. J Clin Oncol 2001;19(2):354-63.

[6] Perez EA, Roche PC, Jenkins RB, et al. HER2 testing in patients with breast cancer: poor correlation between weak positivity by immunohistochemistry and gene amplification by fluorescence in situ hybridization. Mayo Clin Proc 2002;77(2):148-54.

[7] Nassar A, Khoor A, Radhakrishnan R, et al. Correlation of HER2 overexpression with gene amplification and its relation to chromosome 17 aneuploidy: a 5-year experience with invasive ductal and lobular carcinomas. Int J Clin Exp Pathol 2014;7(9):6254-61.

[8] Pauletti G, Dandekar S, Rong H, et al. Assessment of methods for tissue-based detection of the HER-2/neu alteration in human breast cancer: a direct comparison of fluorescence in situ hybridization and immunohistochemistry. J Clin Oncol 2000;18(21):3651-64.

[9] Jacobs TW, Gown AM, Yaziji H, et al. HER-2/neu protein expression in breast cancer evaluated by immunohistochemistry. A study of interlaboratory agreement. Am J Clin Pathol 2000;113(2):251-8.

[10] Kuo SJ, Wang BB, Chang CS, et al. Comparison of immunohistochemical and fluorescence in situ hybridization assessment for HER-2/neu status in Taiwanese breast cancer patients. Taiwan J Obstet Gynecol 2007;46(2):146-51.

[11] Park S, Park HS, Koo JS, et al. Breast cancers presenting luminal B subtype features show higher discordant human epidermal growth factor receptor 2 results between immunohistochemistry and fluorescence in situ hybridization. Cancer 2012;118(4):914-23.

[12] Persons DL, Borelli KA, Hsu PH. Quantitation of HER$2 /$ neu and c-myc gene amplification in breast carcinoma using fluorescence in situ hybridization. Mod Pathol 1997;10(7):720-7. 
[13] Ratcliffe N, Wells W, Wheeler K, et al. The combination of in situ hybridization and immunohistochemical analysis: an evaluation of Her2/neu expression in paraffin-embedded breast carcinomas and adjacent normal-appearing breast epithelium. Mod Pathol 1997;10(12):1247-52.

[14] Jimenez RE, Wallis T, Tabasczka P, et al. Determination of Her-2/Neu status in breast carcinoma: comparative analysis of immunohistochemistry and fluorescent in situ hybridization. Modern Pathology 2000;13(1):3745.

[15] Roses RE, Paulson CE, Sharma A, et al. HER-2/neu overexpression as a predictor for the transition from in situ to invasive breast cancer. Cancer Epidemiol Biomarkers Prev 2009;18(5):1386-9. Published online 2009 Apr 21.
[16] Shomaf M, Masad J, Najjar S, et al. Distribution of breast cancer subtypes among Jordanian women and correlation with histopathological grade: molecular subclassification study. JRSM Short Rep 2013;4(10):2042533313490516. Published online 2013 Sep 13.

[17] Qiao EQ, Ji M, Wu J, et al. Joint detection of multiple immunohistochemical indices and clinical significance in breast cancer. Mol Clin Oncol 2013;1(4):703-10.

[18] Lan C, Liu JM, Liu TW, et al. erb-b2 amplification by fluorescence in situ hybridization in breast cancer specimens read as 2+ in immunohistochemical analysis. Am J Clin Pathol 2005;124(1):97-102. 